\title{
Spherical Collectors Versus Bare Tethers for Drag, Thrust, and Power Generation
}

\author{
Juan R. Sanmartin and Enrico C. Lorenzini
}

\begin{abstract}
Deorbit, power generation, and thrusting performances of a bare thin-tape tether and an insulated tether with a spherical electron collector are compared for typical conditions in low-Earth orbit and common values of length $L=4-20 \mathrm{~km}$ and cross-sectional area of the tether $A=1-5 \mathrm{~mm}^{2}$. The relative performance of moderately large spheres, as compared with bare tapes, improves but still lags as one moves from deorbiting to power generation and to thrusting: Maximum drag in deorbiting requires maximum current and, thus, fully reflects on anodic collection capability, whereas extracting power at a load or using a supply to push current against the motional field requires reduced currents. The relative performance also improves as one moves to smaller $A$, which makes the sphere approach the limiting short-circuit current, and at greater $L$, with the higher bias only affecting moderately the already large bare-tape current. For a 4-m-diameter sphere, relative performances range from 0.09 sphere-to-bare tether drag ratio for $L=4 \mathrm{~km}$ and $A=5 \mathrm{~mm}^{2}$ to 0.82 thrust-efficiency ratio for $L=20 \mathrm{~km}$ and $A=1 \mathrm{~mm}^{2}$. Extremely large spheres collecting the short-circuit current at zero bias at daytime (diameters being about $14 \mathrm{~m}$ for $A=1 \mathrm{~mm}^{2}$ and $31 \mathrm{~m}$ for $A=5 \mathrm{~mm}^{2}$ ) barely outperform the bare tape for $L=4 \mathrm{~km}$ and are still outperformed by the bare tape for $L=20 \mathrm{~km}$ in both deorbiting and power generation; these large spheres perform like the bare tape in thrusting. In no case was sphere or sphere-related hardware taken into account in evaluating system mass, which would have reduced the sphere performances even further.
\end{abstract}

Index Terms-Anodic contactors, electrical propulsion, electrodynamic (ED) space tethers, space power.

\section{INTRODUCTION}

A $\mathrm{N}$ electrodynamic (ED) tether is an orbiting wire or tape exchanging current with the magnetized plasma; the geomagnetic field both induces a motional electric field in the tether and exerts a Lorentz force on its current. A basic issue is the capability to collect electrons from the rarefied ionosphere. Comparing performances in low-Earth orbit (LEO) of ED tethers left bare for electron (anodic) collection [1] and tethers using a large sphere as anodic device, as in the TSS1 and TSS1R missions [2], involves a proper evaluation of anodic collection capabilities. This requires carrying the respective current laws to some common formulation, which is discussed

Manuscript received November 28, 2005; revised May 8, 2006. The work of J. R. Sanmartin was supported by the Spanish Ministerio de Ciencia y Tecnologia under Grant ESP2004-01511.

J. R. Sanmartin is with the School of Aeronautical Engineering, Universidad Politécnica de Madrid, 28040 Madrid, Spain (e-mail: jrs@faia.upm.es).

E. C. Lorenzini is with the Department of Mechanical Engineering, University of Padova, 35100 Padova, Italy, and also with the HarvardSmithsonian Center for Astrophysics, Cambridge, MA 02138 USA (e-mail: enrico.lorenzini@unipd.it).

Digital Object Identifier 10.1109/TPS.2006.883367 in the next section. Independently, gauging performance is different for drag (or deorbit), power generation, and thrust applications and is carried out separately in Sections III-V, respectively. In the tether circuit, we will neglect, in all cases, the small voltage drops through circuit closure in the ionosphere and at the hollow cathode that establishes electrical contact with the ionosphere at the cathodic end. Furthermore, since any tether operates in a slow average way, we will adopt typical ionospheric values in comparing performances while sweeping a broad domain in the geometrical parameters of the tether. Previous comparisons have been very limited in scope [3]-[5].

The bare-tether current will follow the orbital-motion-limited (OML) law for a cylinder, which is applicable to tapes or round wires. For (high) uniform voltage bias $\Phi_{\text {cyl }}$ with respect to the plasma, the current collected per unit length is

$$
\frac{I_{\mathrm{cyl}}(\mathrm{OML})}{L} \approx J_{\mathrm{th}} \times p \times \sqrt{\frac{4}{\pi} \frac{e \Phi_{\mathrm{cyl}}}{k T}} \equiv e N_{\infty} \frac{p}{\pi} \times \sqrt{\frac{2 e \Phi_{\mathrm{cyl}}}{m_{e}}}
$$

where $J_{\text {th }} \equiv e N_{\infty}\left(k T / 2 \pi m_{e}\right)^{1 / 2}$ is the thermal or random current density, $L$ and $p$ are the length and the cross section perimeter of the cylinder, and $T$ and $N_{\infty}$ are the plasma temperature and density, respectively. The magnetic thrust or drag on a tether is $F=\bar{I} L B$, where $B$ is the appropriate magnetic field component and $\bar{I} \equiv \int_{0}^{L} I(s) d s / L$ is the length-averaged current. For a bare tether, the current $I_{\mathrm{bt}}(s)$ will, in general, vary with the position $s$ along the tether both because bias does vary with $s$ and because current piles up. As we shall recall, however, optimal design may require insulating some tether segment, depending on the application (drag, thrust, or power generation).

We shall assume that current to the sphere follows the Parker-Murphy (PM) law [6], which was modified to fit TSS1R results [7]. Here, the length averaged current is just the current to the sphere. Writing

$$
\bar{I}_{\mathrm{sph}}=I_{\mathrm{sph}} \equiv 4 \pi R^{2} \times J_{\mathrm{th}} \times j_{\mathrm{sph}}\left(\Phi_{\mathrm{sph}}\right)
$$

the modified PM law for the dimensionless current is

$$
\begin{gathered}
j_{\mathrm{sph}}=j_{\mathrm{PM}}^{*} \approx a \frac{1+\sqrt{8 \psi}}{2} \\
\psi \equiv \frac{m_{e} \Phi_{\mathrm{sph}}}{e B^{2} R^{2}} \geq 0
\end{gathered}
$$

where $R$ and $\Phi_{\mathrm{sph}}$ are the sphere radius and the (positive) bias. The factor $1 / 2$ describes magnetic guiding of electrons, and $a>1$ is a rough factor fitting TSS1R data. Values $a \approx 2.5$ 
(with the square-root term slightly modified) and 2.16 have been suggested [7]; we shall simply write $a=2$ so as just not to exceed the random current at $\Phi_{\mathrm{sph}}=0$ in an unmagnetized plasma. We found a power law approximation for $j_{\mathrm{PM}}^{*}$ to less than $6 \%$, which eases computations over a $\psi$-range of interest. That is,

$$
j_{\mathrm{PM}}^{*} \approx a \times 2 \psi^{2 / 5} \rightarrow 4 \psi^{2 / 5}, \quad 0.25<\psi<25
$$

and for $B \approx 0.2 \mathrm{G}$ and $\Phi_{\mathrm{sph}} \approx 1.75 \mathrm{kV}, \psi$ lies in the aforementioned range for $R$ in the range of $1-10 \mathrm{~m}$.

\section{NORMALIZED CURRENTS}

Ohmic limitations prove to be critical in comparing bare tethers and TSS1R-like tethers. It is, thus, convenient to normalize currents with the short-circuit value $\sigma_{c} E_{m} A$, which is an upper bound to current in case of drag or power-generation applications; here, $\sigma_{c}, A$, and $E_{m}=U B$ are tether conductivity, crosssectional area, and motional electric field, respectively. We then define dimensionless values as follows:

$$
\begin{gathered}
\bar{i}_{\mathrm{bt}} \equiv \frac{\bar{I}_{\mathrm{bt}}}{\sigma_{c} E_{m} A} \\
\bar{i}_{\mathrm{sph}} \equiv \frac{\bar{I}_{\mathrm{sph}}}{\sigma_{c} E_{m} A} .
\end{gathered}
$$

The magnetic force on the tether now reads $F=\sigma_{c} E_{m} B \times$ $L A \times \bar{i}$. As we shall see, a performance index for each application involves the normalized current $\bar{i}$ and related dimensionless numbers. The force $F$ (and, in some way, the mission itself) is then characterized by tether volume or, equivalently, by mass for a given material. In the following discussion, we shall consider the parametric domain $L=4-20 \mathrm{~km}$ and $A=1-5 \mathrm{~mm}^{2}$, allowing for a range of tether volume $A \times L=0.004-0.1 \mathrm{~m}^{3}$. As regards ionospheric parameters, we use throughout values $E_{m}=150 \mathrm{~V} / \mathrm{km}, k T=0.1 \mathrm{eV}$, and $B=0.2 \mathrm{G}$. However, since density $N_{\infty}$ changes substantially from day to night, we adopt $10^{12} \mathrm{~m}^{-3}$ and $10^{11} \mathrm{~m}^{-3}$ as representative day and night values, respectively. Consequently, the average current, in particular, will be

$$
\bar{i}^{\mathrm{av}} \approx f_{d} \times \bar{i}(\text { day })+\left(1-f_{d}\right) \times \bar{i}(\text { night })
$$

where $f_{d} \approx 0.6$ is the daytime orbit fraction in LEO.

To analyze current and bias profiles along a bare tether, one introduces a length $L^{*}$ defined by the condition that a tether of such length, at uniform bias $E_{m} L^{*}$, collects the short-circuit current (aside from a factor just simplifying dimensionless equations for those profiles) [1]. That is

$$
\begin{aligned}
& \frac{4}{3} e N_{\infty} \frac{p L^{*}}{\pi} \sqrt{\frac{2 e E_{m} L^{*}}{m_{e}}} \equiv \sigma_{c} E_{m} A \\
& L^{*} \propto E_{m}^{1 / 3} \times\left(\frac{\sigma_{c} A}{p N_{\infty}}\right)^{2 / 3} \\
& \Rightarrow \quad L^{*}=0.66 \mathrm{~km} \times\left(\frac{h \times 10^{12} \mathrm{~m}^{-3}}{0.1 \mathrm{~mm} \times N_{\infty}}\right)^{2 / 3}
\end{aligned}
$$

for aluminum; $h \equiv 2 A / p$ is the thickness for thin tapes and radius for round wires. Dimensionless results versus $L^{*} / L$ (and additional dimensionless numbers) will be recalled in Sections III-V [1], [4], [8].

The length $L^{*}$ gauges the bare-tether collection impedance against tether resistance. A small ratio $L^{*} / L$, as with the thin tethers suggested by (7), corresponds to low collection impedance and, as we shall see, to high mass efficiency, because the cross-sectional area $A$ is then fully used to carry current. For a round wire, a value $h=0.1 \mathrm{~mm}$ yields $A \approx 0.03 \mathrm{~mm}^{2}$, resulting in too weak $\mathrm{ED}$ tether forces. In what follows, we consider a tape and set $h=0.1 \mathrm{~mm}$, to yield

$$
\begin{aligned}
L^{*}(\text { day }) & \approx 0.66 \mathrm{~km} \\
L^{*}(\text { night }) & \approx 3.05 \mathrm{~km} .
\end{aligned}
$$

A thickness of $h=0.1 \mathrm{~mm}$ corresponds to an area $A=$ $1-5 \mathrm{~mm}^{2}$ for a tape width $w=10-50 \mathrm{~mm}$. For $A \approx 5 \mathrm{~mm}^{2}$, collection would drop below the OML law at daytime, when the Debye length $\lambda_{D}$ is about $3 \mathrm{~mm}$, well below $w / 4$, which is the radius of an equivalent circular cross section in the OML validity criterion [9]. Current would be reduced below the OML value approximately by a factor of 0.7 [10]. This is equivalent to using a day plasma density $N_{\infty}=0.7 \times 10^{12} \mathrm{~m}^{-3}$; this increases $L^{*}$ (day) by a factor of $0.7^{-2 / 3} \approx 1.27$. No correction is needed at nighttime, i.e., when $\lambda_{D} \sim 9 \mathrm{~mm}$ is close to $w / 4$.

Regarding current to the sphere, we first introduce a characteristic radius defined by the condition that random and shortcircuit currents are equal, i.e.,

$$
\begin{aligned}
4 \pi R^{* 2} J_{\mathrm{th}} & =\sigma_{c} E_{m} A \\
\Rightarrow \quad 10^{-1 / 2} R^{*}(\text { night }) & =R^{*}(\text { day }) \\
& =7.02 \mathrm{~m} \times \sqrt{A\left(\mathrm{~mm}^{2}\right)} .
\end{aligned}
$$

We then use (5b) and (9) to write (2), and (3a) for $\Phi_{\mathrm{sph}}>0$, as

$$
\begin{aligned}
\bar{i}_{\mathrm{sph}} & =\frac{R^{2}}{R^{* 2}} \times j_{\mathrm{sph}}\left(\Phi_{\mathrm{sph}}\right) \\
\bar{i}_{\mathrm{sph}} & =\frac{R^{2}}{R^{* 2}} \times\left(1+\sqrt{K \times \frac{\Phi_{\mathrm{sph}}}{E_{m} L}}\right) \\
K & \approx 17.1 \frac{L(\mathrm{~km})}{[R(\mathrm{~m})]^{2}}
\end{aligned}
$$

with the ratio $\Phi_{\mathrm{sph}} / E_{m} L$ to be obtained from the tether circuit equation. For $2<K \times \Phi_{\mathrm{sph}} / E_{m} L<200$, using (4) yields

$$
\begin{aligned}
\bar{i}_{\mathrm{sph}}= & i^{*} \times\left(\Phi_{\mathrm{sph}} / E_{m} L\right)^{2 / 5} \\
10 i^{*}(\text { night })= & i^{*}(\text { day }) \\
\approx & 0.110[L(\mathrm{~km})]^{2 / 5} \\
& \times[R(\mathrm{~m})]^{6 / 5} / A\left(\mathrm{~mm}^{2}\right) .
\end{aligned}
$$

Clearly, a large enough sphere could make current as large as desired. A basic point of our analysis will be to show how large $R$ must be for a spherical collector to be about as effective as 
a bare tether. We will consider extreme values like $R=2 \mathrm{~m}$, yielding

$$
i^{*}(\mathrm{day}) /[L(\mathrm{~km})]^{2 / 5} \approx 0.051(0.253), \quad \text { for } A=5(1) \mathrm{mm}^{2}
$$

and $R=R^{*}$ (day) $\approx 15.7 \mathrm{~m}$ and $7.02 \mathrm{~m}$ for $A=5$ and $1 \mathrm{~mm}^{2}$, respectively.

\section{DEORBIT Missions}

To gauge performance, we consider the ratio between the total impulse of the deorbiting mission, which is written as the product of drag force $F$ times the mission duration $\tau$, and the mass of the system dedicated to producing the force. That ratio, which should be as large as possible, is the velocity of the exhaust gases (which is proportional to the specific impulse) in case of chemical propulsion where system mass is basically propellant mass. In the case of electrical propulsion, one must also allow for the mass of a power plant. An ED tether needs no power supply for deorbiting and consumes very little (expellant) mass at its hollow cathode. Dedicated mass is then tether mass times some factor $\alpha_{t} \sim 2.5$ that accounts for basic tether-related hardware (deployer/end ballast).

We then have

$$
\frac{\text { Mission impulse }}{\text { Dedicated mass }} \approx \frac{F \tau}{\alpha_{t} \rho A L}=\frac{\sigma_{c} E_{m}^{2} \tau}{\alpha_{t} \rho U} \times \bar{i}^{\mathrm{av}} .
$$

Since all other factors in (14) may have values common to baretether and spherical current collection, performance is measured by the dimensionless average current $\bar{i}^{\text {av }}$, which should be as close as possible to its upper bound (i.e., unity).

For both deorbiting and power generation, the electron current to a bare tether starts from zero at the anodic end at the top (in eastward orbits) and increases downward along the tether as electrons collected under the OML law pile up (Fig. 1). Bias (relative to the local plasma) decreases downward from some positive value at the top, with the drop rate itself decreasing as current increases and vanishing where, and if, the current reaches the short-circuit value $\sigma_{c} E_{m} A$. Since bias must be negative at the bottom to allow a hollow cathode to eject electrons, there exists a point where bias vanishes, with the (cathodic) segment below being at negative bias. For deorbiting, with no load at the bottom, its negative bias is simply equal to the hollow-cathode bias, which is negligibly small; one may, thus, ignore ion collection along the cathodic segment, where current will be uniform [4].

One then finds that for the particular tether length $L=4 L^{*}$, current reaches the short-circuit value at the point of zero bias, whereas, for $L<4 L^{*}$, current remains everywhere below that value; in either case, the cathodic segment is negligibly short. For $L>4 L^{*}$, that segment has the excess length $L-4 L^{*}$ and presents both constant (short-circuit) current and constant (zero) bias. One then has ${ }^{1}$

$$
\bar{i}_{\mathrm{bt}}=1-\varphi_{A}\left(L^{*} / L\right) \times L^{*} / L
$$

\footnotetext{
${ }^{1}$ Equation (15a) is the same as [4, eq. (27)], with bias at the bottom already neglected.
}

\section{Bare tether anode}
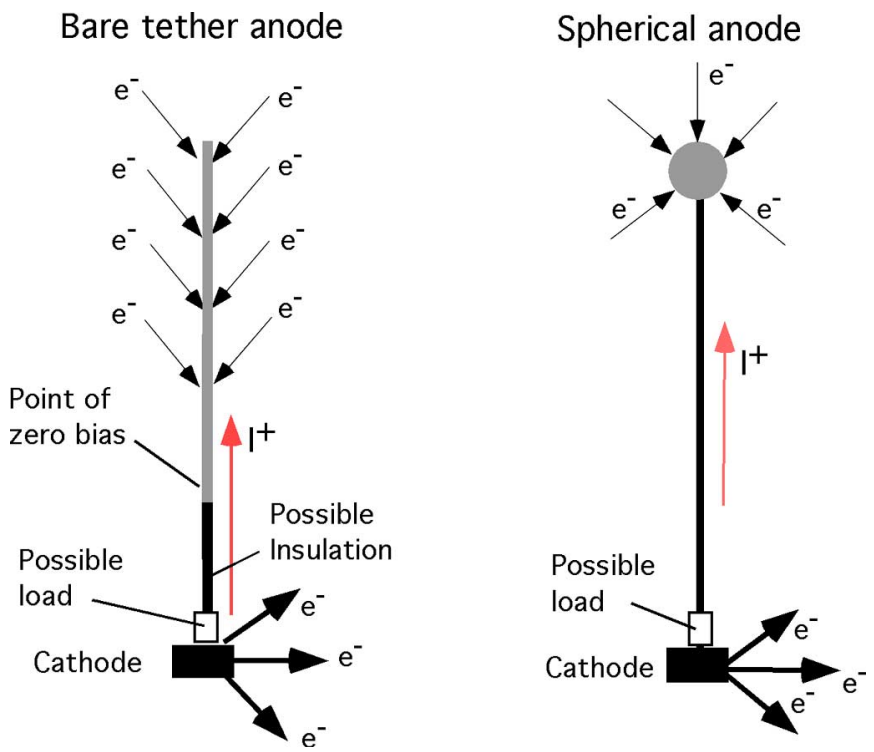

Fig. 1. Sketch of bare-tether and end-sphere anodic collectors for deorbit or power generation. Electrons flow downward (for eastward orbits) and are ejected at a bottom cathodic device. In case of power generation, an electrical load is next to the cathode, and the bare tether has a lower segment insulated to suppress ion collection. (Color version available online at http:// ieeexplore.ieee.org.)

with either of the following equations:

$$
\begin{aligned}
\frac{L}{L^{*}} & =\int_{0}^{\varphi_{A}} \frac{d \varphi}{\sqrt{1-\varphi_{A}^{3 / 2}+\varphi^{3 / 2}}}, \quad L \leq 4 L^{*} \\
\varphi_{A} & =1, \quad L>4 L^{*}
\end{aligned}
$$

with $\varphi_{A}$ being a dimensionless bias at the anodic end. The current $\bar{i}_{\mathrm{bt}}$ is a function of just the tape length $L$ (except for current dropping below the OML value at day for too wide a tape, as already noticed).

For $L=20 \mathrm{~km}$, with $L^{*}$ given in (8), (15c) applies at both day and night, leading to corresponding values $\bar{i}_{\mathrm{bt}} \approx 0.97$ and 0.85 . From (6), we find

$$
\bar{i}_{\mathrm{bt}}^{\mathrm{av}}(20 \mathrm{~km}) \approx 0.97 f_{d}+0.85\left(1-f_{d}\right) \approx 0.92 .
$$

For $L=4 \mathrm{~km},(15 \mathrm{~b})$ and (15c) apply at night and day respectively, finally yielding

$$
\bar{i}_{\mathrm{bt}}^{\mathrm{av}}(4 \mathrm{~km}) \approx 0.84 \times 0.6+0.34 \times 0.4 \approx 0.64 .
$$

Both results are strictly valid for $A=1 \mathrm{~mm}^{2}$; in case $A=$ $5 \mathrm{~mm}^{2}$, the reduction in day current below the OML value is negligible in (16a), but it does change (16b) to $\bar{i}_{\mathrm{bt}}^{\mathrm{av}}$ $(4 \mathrm{~km}) \approx 0.61$.

As regards the sphere, the tether circuit equation would be

$$
E_{m} L=\Phi_{\mathrm{sph}}+Z_{t} I_{\mathrm{sph}}
$$

with $Z_{t}=L / \sigma_{c} A$ being the tether resistance. One readily finds $\Phi_{\mathrm{sph}} / E_{m} L=1-\bar{i}_{\mathrm{sph}}$, (11a) then becoming

$$
\bar{i}_{\mathrm{sph}}=\frac{R^{2}}{R^{*^{2}}} \times\left(1+\sqrt{K \frac{\Phi_{\mathrm{sph}}}{E_{m} L}}\right)=1-\frac{\Phi_{\mathrm{sph}}}{E_{m} L}
$$


TABLE I

Comparison Between Anodic Collection Performances by Bare Tethers and End Spherical Collectors, For a Range of Values in Tether Length $L$, Cross-Sectional Area $A$ (Tape Cross Section of width $=A / 0.1 \mathrm{~mm}$ In Case of Bare Tether), ANd Radius $R$ in Case of a Sphere. Performance Figures of Merit Are (Length-Averaged) Normalized Current for Drag and Maximum Power and Propulsive EfFiciency for Power Generation and Thrust, Respectively. Figures Are Day-Night Averages, Except for Thrust; $\tilde{E}_{m}$ Is a Dimensionless Motional Field as Defined in (41)

\begin{tabular}{|c|c|c|c|c|c|c|c|c|}
\hline$L(\mathrm{~km})$ & \multicolumn{4}{|c|}{20} & \multicolumn{4}{|c|}{4} \\
\hline$A\left(\mathrm{~mm}^{2}\right)$ & \multicolumn{2}{|c|}{5} & \multicolumn{2}{|c|}{1} & \multicolumn{2}{|c|}{5} & \multicolumn{2}{|c|}{1} \\
\hline$R(\mathrm{~m})$ & 2 & 15.7 & 2 & 7.02 & 2 & 15.7 & 2 & 7.02 \\
\hline Drag $i_{b t}^{a v}$ & \multicolumn{4}{|c|}{0.92} & \multicolumn{2}{|c|}{0.61} & \multicolumn{2}{|c|}{0.64} \\
\hline Drag $i_{s p h}^{a v}$ & 0.10 & 0.68 & 0.39 & 0.73 & 0.055 & 0.66 & 0.24 & 0.68 \\
\hline $\begin{array}{l}\text { Power gen. } \\
\operatorname{Max} w_{b t}^{a v}\end{array}$ & \multicolumn{4}{|c|}{0.23} & \multicolumn{4}{|c|}{0.17} \\
\hline $\begin{array}{l}\text { Power gen. } \\
\text { Max } w_{s p h}^{a v}\end{array}$ & 0.041 & 0.20 & 0.13 & 0.20 & 0.023 & 0.19 & 0.09 & 0.20 \\
\hline $\begin{array}{c}\operatorname{Thrust}\left(\widetilde{E}_{m}=1\right) \\
\operatorname{Max} \eta_{b t}{ }^{e f f}\end{array}$ & \multicolumn{4}{|c|}{0.33} & \multicolumn{4}{|c|}{0.33} \\
\hline $\begin{array}{c}\operatorname{Thrust}\left(\widetilde{E}_{m}=1\right) \\
\operatorname{Max} \eta_{s p h} \text { eff }\end{array}$ & 0.13 & 0.33 & 0.27 & 0.33 & 0.088 & 0.33 & 0.21 & 0.33 \\
\hline
\end{tabular}

with $K$ given by (11b). If (11c) applies, one has

$$
\bar{i}_{\mathrm{sph}}=i^{*} \times\left(1-\bar{i}_{\mathrm{sph}}\right)^{2 / 5} .
$$

Consider $R=2 \mathrm{~m}$ and take $A=5 \mathrm{~mm}^{2}$ first. Using $i^{*}$ (night) $=0.1 i^{*}$ (day) and (13), we determine $i^{*}$ at both day and night, and then $\bar{i}_{\mathrm{sph}}$ (day) and $\bar{i}_{\mathrm{sph}}$ (night) from (18b), for both $L=20 \mathrm{~km}$ and $L=4 \mathrm{~km}$. Equation (6) finally yields

$$
\begin{aligned}
\bar{i}_{\mathrm{sph}}^{\mathrm{av}}(L=20 \mathrm{~km}) & \approx 0.10 \\
\bar{i}_{\mathrm{sph}}^{\mathrm{av}}(L=4 \mathrm{~km}) & \approx 0.055 .
\end{aligned}
$$

Similarly, for $R=2 \mathrm{~m}$ and $A=1 \mathrm{~mm}^{2}$, we find

$$
\begin{gathered}
\bar{i}_{\mathrm{sph}}^{\mathrm{av}}(L=20 \mathrm{~km}) \approx 0.39 \\
\bar{i}_{\mathrm{sph}}^{\mathrm{av}}(L=4 \mathrm{~km}) \approx 0.24 .
\end{gathered}
$$

Next, consider $A=5 \mathrm{~mm}^{2}$ and $R=R^{*}$ (day) $\approx 15.7 \mathrm{~m}$. The sphere would now collect the random current at day, with $\Phi_{\mathrm{sph}}=0$ and $\bar{i}_{\mathrm{sph}}=1 \mathrm{in}$ (18a). At night, using $\left[R^{*} \text { (night) }\right]^{2}=$ $10 R^{2}$ and taking the constant $K$ from (11b), we find $\bar{i}_{\mathrm{sph}}($ night $)=0.20(0.15)$ for $L=20 \mathrm{~km}(4 \mathrm{~km})$ from (18a), and finally

$$
\begin{gathered}
\bar{i}_{\mathrm{sph}}^{\mathrm{av}}(L=20 \mathrm{~km}) \approx 0.68 \\
\bar{i}_{\mathrm{sph}}^{\mathrm{av}}(L=4 \mathrm{~km}) \approx 0.66 .
\end{gathered}
$$

Again, for $A=1 \mathrm{~mm}^{2}$ and $R=R^{*}$ (day) $\approx 7.02 \mathrm{~m}$, we find $\bar{i}_{\text {sph }}($ day $)=1$; also, $\bar{i}_{\text {sph }}($ night $)=0.33(0.20)$ for $L=20 \mathrm{~km}$ $(4 \mathrm{~km})$, leading to

$$
\begin{gathered}
\bar{i}_{\mathrm{sph}}^{\mathrm{av}}(L=20 \mathrm{~km}) \approx 0.73 \\
\bar{i}_{\mathrm{sph}}^{\mathrm{av}}(L=4 \mathrm{~km}) \approx 0.68 .
\end{gathered}
$$

Table I collects all the previous results. Sphere currents for $R=2 \mathrm{~m}$ are about $10 \%$ of the corresponding bare-tether values for $A=5 \mathrm{~mm}^{2}$ and $40 \%$ for $A=1 \mathrm{~mm}^{2}$. Increasing $R$ by a factor of about 8 or 4 , respectively, still leaves the sphere current lagging well behind the bare-tether current for $L=20 \mathrm{~km}$ and barely exceeding it for $L=4 \mathrm{~km}$.

\section{Power Generation}

An ED tether generates power at the expense of orbital energy. A tether can thus serve as primary power source only for short times. Performance can be gauged by the ratio between total energy produced (written as mission duration $\tau$ times useful electrical power generated at some efficiency $\eta_{g}$ on an electrical load of $Z_{l}$ impedance) and mass of the system dedicated to generate power

$$
\begin{aligned}
\frac{\text { Generated energy }}{\text { Dedicated mass }} & =\frac{\eta_{g} \times F U \tau}{\alpha_{t} \rho A L} \\
& =\frac{\sigma_{c} E_{m}^{2} \tau}{\alpha_{t} \rho} \times w, \quad\left(w \equiv \bar{i}^{\mathrm{av}} \times \eta_{g}\right) .
\end{aligned}
$$


Performance is here measured by the product $w \equiv \bar{i}^{\text {av }} \times \eta_{g}$, which is the (normalized) power generated per unit system mass.

For power generation, there is an electric load at the bottom (Fig. 1), where the negative bias will be relatively high. In order to suppress ion collection along the cathodic segment of a bare tether, (most of) that segment must be insulated, again, resulting in current keeping constant along it. Both $\bar{i}_{\mathrm{bt}}$ and $\eta_{g}$ and, consequently, $w$, will here be functions of $L^{*} / L$ and of the ratio $Z_{L} / Z_{t}$, which is a free parameter that allows the maximization of power (at a given $L^{*} / L$ ), as usual with generators; note that any dimensionless quantity in the results may be taken as an alternative free parameter. The form of the function $w_{\mathrm{bt}}\left(L^{*} / L, i_{C}\right)$ is particularly simple, with $i_{C}$ being the dimensionless current $I_{C} / \sigma_{c} E_{m} A$ reaching the cathode and load at the bottom of the tether. ${ }^{2}$ That is

$$
\begin{aligned}
w_{\mathrm{bt}} & =i_{C}\left(1-i_{C}\right)\left[1-\xi_{B}\left(i_{C}\right) \times L^{*} / L\right] \\
\xi_{B}\left(\varphi_{A}\right) & \equiv \int_{0}^{\varphi_{A}} \frac{d \varphi}{\sqrt{1-\varphi_{A}^{3 / 2}+\varphi^{3 / 2}}} \\
\varphi_{A}\left(i_{C}\right) & \equiv\left(2 i_{C}-i_{C}^{2}\right)^{2 / 3}
\end{aligned}
$$

where $\xi_{B} \times L^{*}$ is the length from the anodic end to the point of zero bias. From $w_{\mathrm{bt}}\left(L^{*} / L, i_{C}\right)$, one finds the maximum with respect to $i_{C}$ and then a maximum function $\operatorname{Max} w_{\mathrm{bt}}\left(L^{*} / L\right)$. For simplicity, the load impedance $Z_{l}$ is assumed changed from day to night in order to keep at maximum power conditions as density changes. Using a formula similar to (6), i.e.,

$$
w_{\mathrm{bt}}^{\mathrm{av}} \approx f_{d} \times w(\text { day })+\left(1-f_{d}\right) \times w(\text { night })
$$

we finally find

$$
\begin{array}{r}
\operatorname{Max} w_{\mathrm{bt}}^{\mathrm{av}}(L=20 \mathrm{~km})=0.23 \\
\operatorname{Max} w_{\mathrm{bt}}^{\mathrm{av}}(L=4 \mathrm{~km})=0.17 .
\end{array}
$$

For the sphere, the circuit equation would now be

$$
E_{m} L=\Phi_{\mathrm{sph}}+\left(Z_{t}+Z_{l}\right) I_{\mathrm{sph}} .
$$

Using here

$$
\eta_{g}=\frac{Z_{l} I_{\mathrm{sph}}^{2}}{E_{m} L I_{\mathrm{sph}}}=\frac{Z_{l}}{Z_{t}} i_{\mathrm{sph}}
$$

instead of $Z_{l} / Z_{t}$ as free parameter in (27) leads to

$$
\bar{i}_{\mathrm{sph}}=1-\frac{\Phi_{\mathrm{sph}}}{E_{m} L}-\eta_{g}
$$

which must be solved together with (11a). When (11c) is applicable, use of (29) gives

$$
\bar{i}_{\mathrm{sph}}=i^{*}\left(1-\bar{i}_{\mathrm{sph}}-\eta_{g}\right)^{2 / 5} .
$$

\footnotetext{
${ }^{2}$ Equation (24) is the same as [1, eq. (23)].
}

For $R=2 \mathrm{~m}$, (30) does apply, and we have $w_{\mathrm{sph}}\left(i^{*}, \bar{i}_{\mathrm{sph}}\right)$ defined by

$$
w_{\mathrm{sph}}=\bar{i}_{\mathrm{sph}} \times \eta_{g}=\bar{i}_{\mathrm{sph}} \times\left\lfloor 1-\bar{i}_{\mathrm{sph}}-\left(\bar{i}_{\mathrm{sph}} / i^{*}\right)^{5 / 2}\right\rfloor
$$

yielding a maximum function $\operatorname{Max} w_{\mathrm{sph}}\left(i^{*}\right)$, with $i^{*}$ taken from (13), as

$\operatorname{Max} w_{\mathrm{sph}}=\frac{5}{7} \bar{i}_{\mathrm{sph}}-\frac{3}{7} \bar{i}_{\mathrm{sph}}^{2}, \quad \frac{\bar{i}_{\mathrm{sph}}}{\left(1-2 \bar{i}_{\mathrm{sph}}\right)^{2 / 5}}=\left(\frac{2}{7}\right)^{2 / 5} i^{*}$.

Values of Max $w_{\mathrm{sph}}^{\mathrm{av}}$ are also given in Table I for $L=20$ and $4 \mathrm{~km}$, and $A=1$ and $5 \mathrm{~mm}^{2}$.

For $A=5 \mathrm{~mm}^{2}$ and $R=R^{*}$ (day) $\approx 15.7 \mathrm{~m}$, (11a) and (29) would give contradictory (greater than 1 , less than 1 ) values for $\bar{i}_{\mathrm{sph}}$ at day for any positive bias $\Phi_{\mathrm{sph}}$. One must revert to the general expression of (11) for the current, with $R=R^{*}$, using (29) to write

$$
\bar{i}_{\mathrm{sph}}=j_{\mathrm{sph}}\left(\frac{e \Phi_{\mathrm{sph}}}{k T_{e}}\right)=1-\frac{\Phi_{\mathrm{sph}}}{E_{m} L}-\eta_{g}, \quad \Phi_{\mathrm{sph}}<0 .
$$

As with unmagnetized plasmas, $j_{\mathrm{sph}}<1$ values occur at a negative bias $\Phi_{\mathrm{sph}} \sim-k T / e \ll E_{m} L$ [11], with (33) then yielding $\bar{i}_{\text {sph }}($ day $) \approx 1-\eta_{g}$. Hence

$$
\operatorname{Max} w_{\mathrm{sph}}(\text { day }) \approx \operatorname{Max}\left(1-\eta_{g}\right) \times \eta_{g}=0.25 .
$$

At night, $\Phi_{\mathrm{sph}}$ is positive and relatively small. Equations (11a) and (11b) and (29) give $w_{\mathrm{sph}}\left(K, \Phi_{\mathrm{sph}} / E_{m} L\right)$ as

$$
\begin{aligned}
w_{\mathrm{sph}}= & \bar{i}_{\mathrm{sph}} \times \eta_{g} \\
= & 0.1\left(1+\sqrt{\frac{K \Phi_{\mathrm{sph}}}{E_{m} L}}\right) \\
& \times\left[1-0.1\left(1+\sqrt{\frac{K \Phi_{\mathrm{sph}}}{E_{m} L}}\right)-\frac{\Phi_{\mathrm{sph}}}{E_{m} L}\right]
\end{aligned}
$$

yielding maxima $w_{\mathrm{sph}}(K)=0.12$ and 0.11 , and finally, Max $w_{\mathrm{sph}}^{\mathrm{av}}=0.20$ and 0.19 , for $L=20$ and $4 \mathrm{~km}$, respectively. Similar results are found for $A=1 \mathrm{~mm}^{2}$ and $R=R^{*}($ day $) \approx$ $7.02 \mathrm{~m}$.

All the previous results are summarized in Table I. Because performance for power generation is not measured by current, bare-tether and spherical-collector performances are closer than in deorbiting missions. As in the drag case, performance increases with increasing $L$ in either case. For the spherical collector, performance increases with decreasing area $A$; for spheres so large that they would collect the short-circuit current at zero bias, performance is little dependent on $L$ or $A$.

A tether might also provide power for long missions if thrust by a chemical rocket is used to compensate the magnetic drag on the tether. It turns out that for periods longer than 1-2 weeks, with solar power not available, power generation by a ED tether/rocket combination is more efficient as regards fuel consumption than the alternative power source; that is, a fuel cell for direct generation [12]. Note that the magnetic power 
$\dot{m}_{\text {exh }} v_{\text {exh }} U$ (where rocket thrust $\dot{m}_{\text {exh }} v_{\text {exh }}$ is assumed equal to magnetic drag $F$ to keep the orbit stationary) is greater than the rocket output power $1 / 2 \dot{m}_{\mathrm{exh}} v_{\mathrm{exh}}^{2}$. Here, $\dot{m}_{\mathrm{exh}}$ and $v_{\mathrm{exh}}$ are the propellant mass flow rate and velocity at the rocket exhaust. For $\mathrm{LOX}-\mathrm{LH}_{2}$ (specific impulse $\sim 460 \mathrm{~s}$ ), we have $v_{\text {exh }} \sim$ $4.5 \mathrm{~km} / \mathrm{s}<2 U \sim 15 \mathrm{~km} / \mathrm{s}$. The decrease in rocket kinetic energy due to the fuel-mass loss at constant speed $U$ can be shown to exactly make up for the excess over the rocket output [13].

Rocket propellant mass would then contribute to the system mass, with performance now being gauged by the ratio

$$
\frac{\text { Generated energy }}{\text { Dedicated mass }} \approx \frac{\eta_{g} \times F U \tau}{\dot{m}_{\mathrm{exh}} \tau+\alpha_{t} \rho A L} .
$$

Using $\dot{m}_{\mathrm{exh}} v_{\mathrm{exh}}=\bar{I} L B$, we find

$$
\begin{aligned}
& \frac{\text { Generated energy }}{\text { Dedicated mass }}=\frac{\sigma_{c} E_{m}^{2} \tau}{\alpha_{t} \rho} \times \frac{\bar{i}^{\mathrm{av}} \eta_{g}}{1+\bar{i}^{\mathrm{av}} \times \tau / \widetilde{\tau}} \\
& \widetilde{\tau} \equiv \alpha_{t} \rho U v_{\mathrm{exh}} / \sigma_{c} E_{m}^{2} \sim 0.5 \text { weeks }
\end{aligned}
$$

for an aluminum tape and $E_{m} \sim 150 \mathrm{~V} / \mathrm{km}$. In general, both efficiency $\eta_{g}$ and current $\bar{i}^{\text {av }}$ at the maximum value in (37) are functions of the time ratio $\tau / \widetilde{\tau}$; this additional parameter dependence arises from the two-term form of the denominator in (36). Both types of systems would reach a common maximum, with $\eta_{g} \approx 1$, for long enough missions, when the system mass becomes basically propellant mass consumed at the rocket. The bare tether, however, would reach that maximum much earlier.

\section{THRUSTING MisSIONS}

As in the case of deorbiting, performance is gauged by the ratio between total mission impulse and mass of the system dedicated to produce thrust

$$
\begin{aligned}
\frac{\text { Mission impulse }}{\text { Dedicated mass }} & =\frac{F \tau}{\alpha W_{s}+\alpha_{t} \rho A L} \equiv \frac{\tau \eta^{\text {eff }}}{\alpha U} \\
\frac{1}{\eta^{\text {eff }}} & \equiv \frac{W_{s}}{F U} \times\left(1+\frac{\alpha_{t} \rho A L}{\alpha W_{s}}\right)
\end{aligned}
$$

where $W_{s}$ is the supplied power, $\alpha$ is the inverse specific power, and $\eta^{\text {eff }}$ is an overall efficiency that gauges performance, which takes into account the tether-hardware mass as part of the thrusting system. We assume that $\alpha$ is equal to a few tens of kilograms per kilowatt in cases where a dedicated solar power is required, as for example in a "space tug," and less than $10 \mathrm{~kg} / \mathrm{kW}$ in cases where a solar-power system is already in place, as for the reboost of the International Space Station [8]. For simplicity, we ignore the possibility of storing energy in orbit to keep thrusting during eclipses at a mass cost in batteries; limiting ED tether thrusters to daytime operation might actually be desirable [14]. We, thus, set $\tau \rightarrow f_{d} \tau, \bar{i}^{\text {av }}=\bar{i}$ (day), which we shall just write as $\bar{i}$.

In a bare tether acting as thruster, tether voltage increases upward to reach a high bias at the power supply at the top. Electrons collected along the lower anodic segment flow upward to the supply, which takes them to a negative bias to allow emission from the cathode (Fig. 2). A fully bare tether would

\section{Bare tether anode}

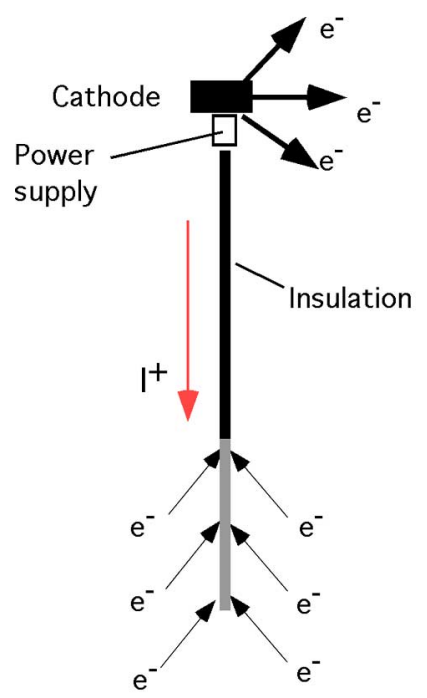

Spherical anode

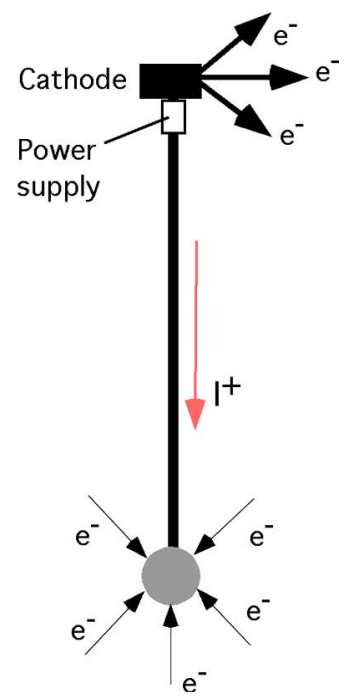

Fig. 2. Sketch as in Fig. 1, for tethers operating as thrusters in eastward orbits. The bare tether has a long upper segment insulated to increase efficiency. (Color version available online at http://ieeexplore.ieee.org.)

be inefficient because electrons collected near the top, acting on a short portion of the tether, would do little push work but would consume full power at the supply when taken across the high bias. For high efficiency, only a lower segment of length $L_{\mathrm{bt}}$ will be left bare. Tether efficiency is then a function of ratios $L^{*} / L, L_{\mathrm{bt}} / L, W_{s} / \sigma_{c} E_{m}^{2} A L$, and a fourth dimensionless number (again, arising from the two-term form of the dedicated mass) that is a normalized value of the motional electric field, with a value of about unity, i.e.,

$$
\widetilde{E}_{m} \equiv E_{m} / \sqrt{\alpha_{t} \rho / \alpha \sigma_{c}} \approx 1.08 \times[\alpha /(10 \mathrm{~kg} / \mathrm{kW})]^{1 / 2} .
$$

Efficiency is highest for low $L^{*} / L$ values [as at daytime operation here, when $L^{*}$ (day) is given by (8a)], with $L_{\mathrm{bt}}$ set to about $L^{*}$ [8]. For both $L^{*} / L$ and $L_{\mathrm{bt}} / L$ very small, $\eta_{\mathrm{bt}}^{\text {eff }}$ takes a simple limiting form as follows ${ }^{3}$ :

$$
\frac{1}{\eta_{\mathrm{bt}}^{\text {eff }}} \equiv 1+\bar{i}_{\mathrm{bt}}+\frac{1}{\widetilde{E}_{m}^{2} \bar{i}_{\mathrm{bt}}}
$$

where the current $\bar{i}_{\mathrm{bt}}$ instead of $W_{s} / \sigma_{c} E_{m}^{2} A L$ is used as a free parameter to further maximize efficiency. This situation corresponds to a negligible anodic impedance in the tether current loop as

$$
W_{s} / \bar{I}_{\mathrm{bt}}=\varepsilon_{s}=E_{m} L+\bar{I}_{\mathrm{bt}} Z_{t}
$$

where $\varepsilon_{s}$ is the supply voltage. The efficiency $\eta_{\mathrm{bt}}^{\text {eff }}\left(\widetilde{E}_{m}, \bar{i}_{\mathrm{bt}}\right)$ in (42) has a maximum given by

$$
\frac{1}{\operatorname{Max} \eta_{\mathrm{bt}}^{\text {eff }}}=1+\frac{2}{\widetilde{E}_{m}}
$$

for $W_{s}$ selected in such a way that $\bar{i}_{\mathrm{bt}}=1 / \widetilde{E}_{m}$.

\footnotetext{
${ }^{3}$ Equation (41) corresponds to [8, eqs.(19), (20a), and (20b)], and (42) corresponds to [8, eq. (24)].
} 
For the tether with a sphere, one readily finds

$$
W_{s} / \bar{I}_{\mathrm{sph}}=\varepsilon_{s}=\Phi_{\mathrm{sph}}+E_{m} L+\bar{I}_{\mathrm{sph}} Z_{t} .
$$

For $R=2 \mathrm{~m}$, we use (11c), which is written as

$$
\Phi_{\mathrm{sph}}=E_{m} L \times\left[\bar{i}_{\mathrm{sph}} / i^{*}\right]^{5 / 2}
$$

in (45) to yield

$$
\frac{1}{\eta_{\mathrm{sph}}^{\mathrm{eff}}} \approx \frac{\bar{i}_{\mathrm{sph}}^{5 / 2}}{i^{*}(\text { day })^{5 / 2}}+1+\bar{i}_{\mathrm{sph}}+\frac{1}{\widetilde{E}_{m}^{2} \bar{i}_{\mathrm{sph}}} .
$$

The efficiency $\left.\eta_{\mathrm{sph}}^{\mathrm{eff}}\left[\widetilde{E}_{m}, i^{*}(\mathrm{day}), \bar{i}_{\mathrm{sph}}\right]\right)$ has a maximum $\operatorname{Max} \eta_{\mathrm{sph}}^{\mathrm{eff}}\left[\widetilde{E}_{m}, i^{*}(\right.$ day $\left.)\right]$ and is given by

$$
\begin{aligned}
\frac{1}{\operatorname{Max} \eta_{\mathrm{sph}}^{\mathrm{eff}}}=1+ & \frac{3}{5} \bar{i}_{\mathrm{sph}}+\frac{7 / 5}{\widetilde{E}_{m}^{2} \bar{i}_{\mathrm{sph}}} \\
& \text { at } \frac{5}{2} \times\left[\frac{\bar{i}_{\mathrm{sph}}}{i^{*}(\text { day })}\right]^{5 / 2}=\frac{1-\widetilde{E}_{m}^{2} \bar{i}_{\mathrm{sph}}^{2}}{\widetilde{E}_{m}^{2} \bar{i}_{\mathrm{sph}}} .
\end{aligned}
$$

Table I presents values of $\eta_{\mathrm{bt}}^{\text {eff }}$ and $\eta_{\mathrm{sph}}^{\text {eff }}$ at $\widetilde{E}_{m}=1$ for $R=$ $2 \mathrm{~m}, A=5$ and $1 \mathrm{~mm}^{2}$, and $L=20$ and $4 \mathrm{~km}$, as given by (44) and (48).

Finally, assuming $A=5 \mathrm{~mm}^{2}$ and $R=R^{*}$ (day) $=15.7 \mathrm{~m}$, or $A=1 \mathrm{~mm}^{2}$ and $R=R^{*}$ (day) $=7.02 \mathrm{~m}$, we have, from (11), $\bar{i}_{\mathrm{sph}}=j_{\mathrm{sph}}\left(\Phi_{\mathrm{sph}}\right)$. For $\widetilde{E}_{m}=1$, under the ansatz $\bar{i}_{\mathrm{sph}}=$ 1 , we would have $\Phi_{\mathrm{sph}}=0$ (zero anodic impedance), (45) transforming into (43) for the bare tether. An equation similar to (42) then yields $1 / \mathrm{Max} \eta_{\mathrm{sph}}^{\mathrm{eff}}=3$ at $\bar{i}_{\mathrm{sph}}=1 / \widetilde{E}_{m}=1$, satisfying the ansatz.

\section{CONCLUSION}

We have compared maximum performances of bare thin-tape tethers and insulated tethers with spherical collector in the three basic applications of power generation, dragging, and thrusting for common values of length $L=4-20 \mathrm{~km}$, cross-sectional area $A=1-5 \mathrm{~mm}^{2}$, and typical conditions in LEO. For moderately large spheres, the relative performance of the insulated tether, when compared with the bare tether, improves but still lags as one moves from deorbiting to power generation and to thrusting. Maximum drag in deorbiting requires maximum current and, thus, fully reflects on anodic collection capability, whereas extracting power at a load, or using a power supply to push current against the motional electric field, requires reduced currents. The relative performance also improves as one moves to smaller $A$, which makes the sphere approach the limiting short-circuit current, and at greater values of $L$, the higher bias affecting only moderately the already large baretape current. For a 4-m diameter, our results show relative performances ranging from 0.09 sphere-to-bare tether drag ratio for $L=4 \mathrm{~km}$ and $A=5 \mathrm{~mm}^{2}$ to 0.82 thrust-efficiency ratio for $L=20 \mathrm{~km}$ and $A=1 \mathrm{~mm}^{2}$. Very large spheres collecting the short-circuit current at zero bias at daytime (diameters being about $14 \mathrm{~m}$ for $A=1 \mathrm{~mm}^{2}$ and $31 \mathrm{~m}$ for $A=5 \mathrm{~mm}^{2}$ ) barely outperform the bare tape for $L=4 \mathrm{~km}$ and are still outper- formed for $L=20 \mathrm{~km}$ in both dragging and power generation; these spheres perform like a bare tape in thrusting. In no case was the sphere or sphere-related hardware taken into account in evaluating the system mass, which would have reduced the sphere performances even further.

\section{REFERENCES}

[1] J. R. Sanmartin, M. Martinez-Sanchez, and E. Ahedo, "Bare wire anodes for electrodynamic tethers," J. Propuls. Power, vol. 9, no. 3, pp. 353-360, Jun. 1993.

[2] M. Dobrowolny and N. H. Stone, "A technical overview of TSS-1: The first tethered-satellite system mission," Il Nuovo Cimento, vol. 17, no. 1, pp. 1-12, 1994.

[3] G. Vannaroni, M. Dobrowolny, and F. De Venuto, "Deorbiting of LEO satellites with electrodynamic tethers," presented at the 38th Aerospace Sciences Meeting and Exhibit, Reno, NV, Jan. 10-13, 2000, AIAA Paper 2000-0328.

[4] E. Ahedo and J. R. Sanmartin, "Analysis of bare-tether systems for deorbiting low-Earth-orbit satellites," J. Spacecr. Rockets, vol. 39, no. 2, pp. 198-205, 2002.

[5] N. H. Stone, J. D. Moore, W. R. Clayton, and P. A. Gierow, "A preliminary assessment of grid spheres used as end-body electrodes for electrodynamic tethers," in Proc. Space Technol. and Appl. Int. Forum, M. S. El-Genk, Ed., New York, 2002, pp. 537-547.

[6] L. W. Parker and B. L. Murphy, "Potential buildup on an electron-emitting ionospheric satellite," J. Geophys. Res., vol. 72, no. 5, pp. 1631-1636, 1967.

[7] D. C. Thompson, C. Bonifazi, B. E. Gilchrist, S. D. Williams, W. J. Raitt, J. P. Lebreton, W. J. Burke, N. H. Stone, and K. H. Wright, Jr., "The current-voltage characteristics of a large probe in low Earth orbit: TSS-1R results," Geophys. Res. Lett., vol. 25, no. 4, pp. 413-416, 1998.

[8] J. R. Sanmartin, R. D. Estes, E. C. Lorenzini, and S. A. Elaskar, "The efficiency of electrodynamic tether thrusters," J. Spacecr. Rockets, vol. 43, no. 3, pp. 659-666, 2006.

[9] J. R. Sanmartin and R. D. Estes, "The orbital-motion-limited regime of cylindrical Langmuir probes," Phys. Plasmas, vol. 6, no. 1, pp. 395-405, Jan. 1999.

[10] R. D. Estes and J. R. Sanmartin, "Cylindrical Langmuir probes beyond the orbital-motion-limited regime," Phys. Plasmas, vol. 7, no. 10, pp. $4320-4325$, Oct. 2000.

[11] J. G. Laframboise and L. J. Sonmor, "Current collection by probes and electrodes in space magnetoplasmas: A review," J. Geophys. Res., vol. 98, no. A1, pp. 337-357, Jan. 1993.

[12] M. Martinez-Sanchez and D. E. Hastings, "A systems study of a $100 \mathrm{~kW}$ electrodynamic tether," J. Astronaut. Sci., vol. 35, no. 1, pp. 7596, Jan.-Mar. 1987.

[13] J. R. Sanmartin, "Physics and applications of electrodynamic space tethers," in Simplicity, Rigor and Relevance in Fluid Mechanics, A Volume in Honor of Amable Liñan. Barcelona, Spain: CIMNE, 2004, pp. 316-330.

[14] J. Pearson, J. Carroll, E. Levin, J. Oldson, and P. Hausgen, "Overview of the electrodynamic delivery express (EDDE)," presented at the 39th Joint Propulsion Conf. and Exhibit, Huntsville, AL, Jul. 20-23, 2003, AIAA Paper 2003-4790.

Juan R. Sanmartin, photograph and biography not available at the time of publication.

Enrico C. Lorenzini, photograph and biography not available at the time of publication. 